\title{
MULTIPLIERS FOR CERTAIN CONVOLUTION MEASURE ALGEBRAS
}

\author{
BY \\ CHARLES DWIGHT LAHR
}

\begin{abstract}
Let $(A, *)$ be a commutative semisimple convolution measure algebra with structure semigroup $\Gamma$, and let $S$ denote a commutative locally compact topological semigroup. Under the assumption that $A$ possesses a weak bounded approximate identity, it is shown that there is a topological embedding of the multiplier algebra $M(A)$ of $A$ in $M(\Gamma)$. This representation leads to a proof of the commutative case of Wendel's theorem for $A=L_{1}(G)$, where $G$ is a commutative locally compact topological group. It is also proved that if $l_{1}(S)$ has a weak bounded approximate identity of norm one, then $M\left(l_{1}(S)\right)$ is isometrically isomorphic to $l_{1}(\Omega(S))$, where $\Omega(S)$ is the multiplier semigroup of $S$. Likewise, if $S$ is cancellative, then $\mathcal{C}\left(l_{1}(S)\right)$ is isometrically isomorphic to $l_{1}(\Omega(S))$.

An example is provided of a semigroup $S$ for which $l_{1}(\Omega(S))$ is isomorphic to a proper subset of $\mathcal{O}\left(l_{1}(S)\right)$.
\end{abstract}

1. Introduction. Let $(A, *)$ be a commutative semisimple convolution measure algebra with structure semigroup $\Gamma$, and let $S$ denote a commutative locally compact topological semigroup with multiplier semigroup $\Omega(S)$ (details concerning notation, definitions, and background results are given in $\$ 2)$. In $\$ 3$ we study the multiplier algebra $\mathcal{C}(A)$ of $(A, *)$ when $A$ possesses a weak bounded approximate identity. Theorem 3.1 asserts that $M(A)$ is topologically embeddable in $M(\Gamma)$. We apply Theorem 3.1 to the particular convolution measure algebra $A=M(S)$ and proceed in Corollary 3.8 to characterize those measures in $M(\Gamma)$ that give rise to multipliers of $M(S)$. Finally, $\$ 4$ is devoted to a characterization of the multiplier algebra of $l_{1}(S)$ for certain semigroups $S$. An example is furnished of a semigroup $S$ for which $l_{1}(\Omega(S))$ is isomorphic to a proper subset of $\mathcal{C}\left(l_{1}(S)\right)$.

2. Preliminaries. Let $(B, *)$ be a commutative Banach algebra under $\|\cdot\|$. Let $\Delta(B)$ denote the maximal ideal space of $B$, that is, the space of all continuous homomorphisms of $B$ into the complex field $C$ together with the weak-* (Gelfand) topology [17]. As usual for any $\alpha \in B$, define $\hat{\alpha}(\chi)=\chi(\alpha)$ for each $\chi \in \Delta(B)$, and let $\hat{B}=\{\hat{\alpha}: \alpha \in B\}$. A weak bounded approximate identity of norm $R$ for $B$ is a net $\left\{E_{\rho}\right\}_{\rho \in g}$ of elements of $B$ such that (a) $\left\|E_{\rho}\right\| \leq R$ for some natural number $R$ and for all $\rho \in \mathcal{Y}$, and (b) $\left(\alpha * E_{\rho}\right)^{\wedge}(\chi) \rightarrow \hat{\alpha}(\chi)$ for all $\chi \in \Delta(B)$ and for every $\alpha \in B$. A bounded approximate identity of norm $R$ for $B$ is a net $\left\{E_{\rho}\right\}$ of elements of $B$ such that (a) $\left\|E_{\rho}\right\| \leq R$ for some natural number $R$ and for all $\rho$, and (b) $\left\|\alpha * E_{\rho}-\alpha\right\| \rightarrow 0$ for all $\alpha \in B$; we sometimes use the

Presented to the Society, January 17, 1972 under the title Approximate identities and multipliers for certain convolution measure algebras; received by the editors March 22, 1972.

AMS (MOS) subject classifications (1970). Primary 43A10, 43A20.

Key words and phrases. Convolution measure algebra, $l_{1}$-algebra, multiplier. 
terminology "bounded (norm) approximate identity of norm $R$ " for the same concept.

If $X$ is any normed linear vector space, the continuous linear dual of $X$ is denoted by $X^{*} ;(\alpha, f)$ represents the action of $f \in X^{*}$ on $\alpha \in X$; and if $Y \subseteq X$, $Z \subseteq X^{*}$, then let $w(Y, Z)$ be the weak topology on $Y \subseteq X$ induced by $Z \subseteq X^{*}$. The natural mapping of $X$ into $X^{* *}$ is denoted by $j: X \rightarrow X^{* *}$. Often, we simply denote $(f, j(\alpha))$ by $(\alpha, f), \alpha \in X, f \in X^{*}$, in those circumstances where the meaning is clear.

A bounded linear operator $T$ from $B$ to $B$ is called a multiplier of $B$ if $T(\alpha * \beta)=\alpha * T(\beta)$ for all $\alpha, \beta \in B$. The set of multipliers of $B$, in turn, forms a Banach algebra of operators under operator norm $|\|\cdot\||$; denote this algebra by $\mathcal{C}(B)$. $M(B)$ contains an identity, and if $B$ is semisimple, it contains an isomorphic copy of $B$ as an ideal. If $B$ has an identity $E$, then each $T \in \mathcal{M}(B)$ is given by multiplication by the fixed element $T(E)$ of $B$ and so $M(B)$ reduces to $B$. If $B$ is semisimple, then $M(B)$ is semisimple, and $\Delta(B)$ is homeomorphic to an open subset of $\Delta(\mathcal{C}(B))$, both in their Gelfand topologies. For a detailed review of results on multipliers, see [13]; also consult [21] and [2].

Now let $S$ be a commutative locally compact Hausdorff semigroup with jointly continuous multiplication (sometimes herein referred to as a commutative locally compact topological semigroup), and let $M(S)$ denote the complex Banach algebra of all bounded regular Borel measures on $S$ where the product * is defined by convolution. For $\mu, \nu \in M(S), F$ a Borel subset of $S,(\mu * \nu)(F)$ $=\int_{S} \int_{S} \phi_{F}(x y) d \mu(x) d \nu(y)$, where $\phi_{F}$ denotes the characteristic function of $F$. The norm on $M(S)$ is the total variation norm, denoted $\|\cdot\|$. See Taylor [20]. A semicharacter $\chi$ on $S$ is a nonzero continuous complex valued function on $S$ of modulus less than or equal to one which satisfies $\chi(x y)=\chi(x) \chi(y)$ for all $x$, $y \in S$. The collection of semicharacters of $S$ is denoted by $\hat{S}$. It is well known that $C_{0}(S)^{*}=M(S)$, where $\mu \in M(S)$ induces a linear functional on $C_{0}(S)$ by

$$
(g, \mu)=\int_{S} g(x) d \mu(x) \text { for all } g \in C_{0}(S) .
$$

The set of discrete measures in $M(S)$ forms a subalgebra of $M(S)$, denoted by $l_{1}(S)$. Of course, if $S$ is discrete, then $l_{1}(S)=M(S)$. Hewitt and Zuckerman present a detailed study of $l_{1}(S)$ in [10]. They show that the existence of an identity in $l_{1}(S)$ is equivalent to the existence of a finite set of relative units in $S$, where $U$ is defined to be a set of relative units for $S$, if for every $x \in S$, there exists $u \in U$ such that $x u=x$. Lardy [12] proves that the same conditions on $S$ are necessary and sufficient for the existence of an identity in $M(S)$, and in fact an identity for $M(S)$ must lie in $l_{1}(S)$.

A portion of this paper is devoted to characterizing the multiplier algebras of certain semisimple convolution measure algebras. For a definition of convolution measure algebra see [20]; $M(S)$ is an example of a convolution measure algebra. Also, if $G$ is a locally compact abelian topological group, and if $L_{1}(G)$ is the 
algebra of Haar integrable functions on $G$ under convolution multiplication, then $L_{1}(G)$ is a convolution measure algebra.

Taylor proves in [20] that if $(A, *)$ is a commutative convolution measure algebra, we may identify the maximal ideal space of $(A, *)$ with $\hat{\Gamma}$, the set of all semicharacters on a compact topological semigroup $\Gamma$, which he labels the structure semigroup of $(A, *)$ ( $\Gamma$ will denote the structure semigroup of whatever convolution measure algebra is under discussion). There is a homomorphism $p: \mu \rightarrow \mu_{p}$ of $A$ into $M(\Gamma)$ with the following pertinent properties: $p(A)$ is weak-* dense in $M(\Gamma)$, that is, dense in the $w(M(\Gamma), C(\Gamma))$ topology (where we have identified $C(\Gamma)$ with its natural image in $\left.M(\Gamma)^{*}\right) ; p$ is an isometry if and only if $(A, *)$ is semisimple. $\Gamma$ also has the property that the uniformly closed linear span of $\hat{\Gamma}$ is $C(\Gamma)$. We make use of this fact in observing that $\hat{\Gamma} \subseteq \Delta(M(\Gamma))$ is enough to decide the semisimplicity of $M(\Gamma)$ : that is, suppose $\mu, \nu \in M(\Gamma)$ and $\hat{\mu}(\chi)$ $=\hat{\nu}(\chi)$ for all $\chi \in \hat{\Gamma}$; then because the linear span of $\hat{\Gamma}$ is uniformly dense in $C(\Gamma)$, the formula $(\chi, \mu)=(\chi, \nu)$ for all $\chi \in \hat{\Gamma}$ can be extended to all $f \in C(\Gamma)$; therefore, $\mu$ and $\nu$ agree as linear functionals on $C(\Gamma)$ and so as elements of $M(\Gamma)$.

Suppose $(A, *)$ is semisimple. Then it is proved in [11] that $A$ has a weak bounded approximate identity if and only if $\Gamma$ has a finite set of relative units. In fact, the existence of an identity in $\Gamma$ is equivalent to the existence of a weak bounded approximate identity of norm one in $A$.

3. Multipliers of convolution measure algebras. Throughout this section $(A, *)$ is a semisimple convolution measure algebra, $S$ is a locally compact abelian Hausdorff semigroup with jointly continuous multiplication, and $M(S)$ is assumed to be semisimple. The following is a representation theorem for $M(A)$.

Theorem 3.1. Let $\left\{E_{\rho}\right\}$ be a weak bounded approximate identity for $(A, *)$, $\left\|E_{\rho}\right\| \leq R$ for all $\rho$. Then if $T \in \mathcal{M}(A)$ there is a unique measure $\mu_{T} \in M(\Gamma)$ such that $T(\alpha)=\alpha * \mu_{T}$ for all $\alpha \in A$ and $\mathcal{M}(A)$ is isomorphic to $\{\mu \in M(\Gamma): \mu * \alpha$ $\in A$ for all $\alpha \in A$ \}. Furthermore, the correspondence $T \mapsto \mu_{T}$ is an isometry if and only if $A$ has a weak bounded approximate identity of norm one; in any case \|\|$T\|\leq\| \mu_{T}\|\leq R\| T\|\|$.

Proof. Assume $A$ is embedded in $M(\Gamma)$. Suppose $T \in \mathcal{M}(A)$. Then $\left\{T\left(E_{\rho}\right)\right\}$ is a subset of the closed ball of $M(\Gamma)$ of radius $R|\|T\||$. In the weak-* topology this ball is compact. Thus, there is a subnet $\left\{T\left(E_{\rho^{\prime}}\right)\right\}$ and $\mu_{T} \in M(\Gamma)$ such that $\mu_{T}$ is a weak-* limit of $\left\{T\left(E_{\rho^{\prime}}\right)\right\}$; hence, for all $f \in C(\Gamma),\left(f, T\left(E_{\rho^{\prime}}\right)\right) \rightarrow\left(f, \mu_{T}\right)$ and in particular $\left(\chi, T\left(E_{\rho^{\prime}}\right)\right) \rightarrow\left(\chi, \mu_{T}\right)$ for all $\chi \in \hat{\Gamma}$. If $\alpha \in A,\left(\chi, T\left(E_{\rho^{\prime}}\right)\right)(\chi, \alpha) \rightarrow$ $\left(\chi, \mu_{T}\right)(\chi, \alpha)$ and so $\left(\chi, \alpha * T\left(E_{\rho^{\prime}}\right)\right) \rightarrow\left(\chi, \alpha * \mu_{T}\right)$ for all $\chi \in \hat{\Gamma}$. On the other hand, since $\left\{E_{\rho}\right\}$ is a weak bounded approximate identity, $\left(\chi, \alpha * T\left(E_{\rho}\right)\right)=(\chi, T(\alpha)$ $\left.* E_{\rho}\right) \rightarrow(\chi, T(\alpha))$ for all $\chi \in \hat{\Gamma}$ and for all $\alpha \in A$. Thus, $(\chi, T(\alpha))=\left(\chi, \alpha * \mu_{T}\right)$ for all $\chi \in \hat{\Gamma}$. Because the linear span of $\hat{\Gamma}$ is uniformly dense in $C(\Gamma)$, this formula can be extended to all $f \in C(\Gamma)$. Therefore, $T(\alpha)$ and $\mu_{T} * \alpha$ agree as linear functionals on $C(\Gamma)$ and so as elements of $M(\Gamma)$. 
To see that $\mu_{T}$ is unique, suppose $\mu, \lambda \in M(\Gamma)$ and $\mu * \alpha=\lambda * \alpha$ for all $\alpha \in A$. If $\chi \in \hat{\Gamma}$, there is an $\alpha \in A$ such that $(\chi, \alpha) \neq 0$. Thus, $(\chi, \mu-\lambda)(\chi, \alpha)=0$ implies that $(\chi, \mu-\lambda)=0$. Since this is true for all $\chi \in \hat{\Gamma}$ and the linear span of $\hat{\Gamma}$ is dense in $C(\Gamma),(f, \mu-\lambda)=0$ for all $f \in C(\Gamma)$. Thus, $\mu-\lambda=0$ or $\mu=\lambda$. These arguments show that $\mathcal{} M(A)$ is isomorphic to $\{\mu \in M(\Gamma): \mu * \alpha \in A$ for all $\alpha \in A\}$.

Now, since $\mu_{T}$ is a weak-* limit point of a net in the closed ball of radius $R\|T\|$, $\left\|\mu_{T}\right\| \leq R\|T\|$, so the mapping $T \mapsto \mu_{T}$ is continuous. Also, $T(\alpha)$ $=\alpha * \mu_{T}$ for all $\alpha \in A$ implies that $\|T(\alpha)\| \leq\left\|\mu_{T}\right\|\|\alpha\|$ and hence $\|T\|\|\leq\| \mu_{T} \|$. Thus, $\|\cdot \cdot\| \mid$ on $\mathcal{M}(A)$ is equivalent to $\|\cdot\|$ on $\{\mu \in M(\Gamma): \mu * \alpha \in A$ for all $\alpha \in A$ \}. From the relationship $|\|T\|| \leq\left\|\mu_{T}\right\| \leq R\|T\| \mid$ we see that if $R=1$, $T \mapsto \mu_{T}$ is actually an isometry.

On the other hand, if the mapping $T \mapsto \mu_{T}$ is an isometry, let $I$ denote the identity multiplier and $\mu_{I}$ the corresponding measure in $M(\Gamma)$ such that $\alpha=I(\alpha)$ $=\alpha * \mu_{1}$ for all $\alpha \in A$. Then, because $A$ is weak- dense in $M(\Gamma), \mu_{I}$ is the identity for $M(\Gamma)$ : let $\mu \in M(\Gamma)$; then there exists a net $\left\{\mu_{d}\right\} \subset A$ such that $\mu_{d} \rightarrow \mu$ weak-*; of course, $\mu_{d} * \mu_{l}=\mu_{d}$ for all $d$, and hence

$$
\left(\chi, \mu_{d}\right)=\left(\chi, \mu_{d} * \mu_{l}\right)=\left(\chi, \mu_{d}\right)\left(\chi, \mu_{l}\right) \rightarrow(\chi, \mu)\left(\chi, \mu_{I}\right)=\left(\chi, \mu * \mu_{l}\right)
$$

for all $\chi \in \hat{\Gamma}$; thus, $(\chi, \mu)=\left(\chi, \mu * \mu_{l}\right)$ for all $\chi \in \hat{\Gamma}$ and so $\mu=\mu * \mu_{1}$. Therefore, $\mu_{l} \in l_{1}(\Gamma)[12]$, and in fact $\mu_{I}$ is the Hewitt and Zuckerman identity for $l_{1}(\Gamma)$ constructed from the finite set of relative units of $\Gamma[12],[10]$. If $\left\|\mu_{I}\right\|=|\|I\||$ $=1$, it must be that $\mu_{I}$ is a unit point mass concentrated at, say, $e \in \Gamma$. Thus, $e$ is the identity for $\Gamma$ and so $A$ must have a weak bounded approximate identity of norm one by Corollary 3.2 of [11]. This completes the proof.

In Theorem 3.1 of [11] it is proved that $A$ has a weak bounded approximate identity if and only if $M(\Gamma)$ has an identity. This means that $M(A)$ will not have a representation as a subalgebra of $M(\Gamma)$ unless $A$ has a weak bounded approximate identity as in Theorem 3.1.

If $G$ is a locally compact abelian topological group, the structure semigroup corresponding to $L_{1}(G)$ under convolution product * is $\bar{G}$, the Bohr compactifcation of $G$ [20]. Since $L_{1}(G)$ is a semisimple convolution measure algebra having a bounded approximate identity of norm one, it follows from Theorem 3.1 that if $T \in \mathcal{C}\left(L_{1}(G)\right)$, there exists a measure $\mu_{T} \in M(\bar{G})$ such that $\|T\|=\left\|\mu_{T}\right\|$ and $T(\alpha)=\mu_{T} * \alpha$ for all $\alpha \in L_{1}(G)$. In the next theorem we demonstrate that $\mu_{T}$ is supported on $G$; that is, $\mu_{T} \in M(G)$. In this way Theorem 3.1 can be viewed as a generalization of Wendel's result [22] which identifies $M\left(L_{1}(G)\right)$, isomorphically and isometrically, as $M(G)$.

Theorem 3.2 (Wendel). If $G$ is a locally compact abelian topological group, $M\left(L_{1}(G)\right)$ is isometrically isomorphic to $M(G)$.

Proof. Let $T \in \mathcal{C}\left(L_{1}(G)\right)$. By Theorem 3.1 there is a unique measure $\mu_{T} \in M(\bar{G})$ such that $\|T\|=\left\|\mu_{T}\right\|$ and $T(\alpha)=\mu_{T} * \alpha$ for all $\alpha \in L_{1}(G)$, where 
$\bar{G}$ is the Bohr compactification of $G$. To see that $\mu_{T}$ is concentrated on $G$, observe first that $\hat{\mu}_{T}$ is a continuous function on $\hat{G}$, i.e., it is $w\left(\hat{G}, L_{1}(G)\right)$ continuous. Next note that since $G$ is dense in $\bar{G}$ each $\chi \in \hat{G}$ extends to a character on $\bar{G}$; in like manner, if $f(x)=\sum_{i=1}^{n} c_{i} \chi_{i}(x), \chi_{i} \in \hat{G}, c_{i} \in C$ for $i=1,2, \ldots, n$, is a trigonometric polynomial on $G, f$ can be extended to a trigonometric polynomial on $\bar{G}$ without increase in norm and

$$
\begin{aligned}
\left|\sum_{i=1}^{n} c_{i} \hat{\mu}_{T}\left(x_{i}\right)\right| & =\left|\sum_{i=1}^{n} c_{i}\left(\mu_{T}, x_{i}\right)\right| \\
& =\left|\sum_{i=1}^{n} c_{i} \int_{\bar{G}} x_{i}(\bar{x}) d \mu_{T}(\bar{x})\right| \\
& =\left|\int_{\bar{G}} f(\bar{x}) d \mu_{T}(\bar{x})\right| \leq\left\|\mu_{T}\right\|\|f\|_{\infty} .
\end{aligned}
$$

Now, following Rudin's proof of Eberlein's theorem [18, p. 32], there is a measure $\mu \in M(\bar{G})$ such that

$$
\hat{\mu}_{T}(\chi)=\int_{\bar{G}} \chi(\bar{x}) d \mu(\bar{x}), \quad \chi \in \hat{G},
$$

and in fact $\mu$ is actually concentrated on $G . \hat{\mu}_{T}(\chi)=\hat{\mu}(\chi)$ for all $\chi \in \hat{G}$ implies $\mu_{T} \equiv \mu$, and hence $\mu_{T}$ is concentrated on $G$.

Conversely, it is well known that all elements of $M(G)$ are multipliers of $L_{1}(G)$. This completes the proof.

Theorem 3.1 gives a characterization of $M(M(S))$ when $M(S)$ has a weak bounded approximate identity. Using the approach of Rennison [16] in applying Arens product [1] techniques to the task of describing the structure semigroup $\Gamma$ corresponding to $(M(S), *)$, it is possible to better identify those measures in $M(\Gamma)$ that give rise to multipliers of $M(S)$. Whenever no ambiguity arises, we denote the uniformly closed subspace of $M(S)^{*}$ generated by $\Delta(M(S))$ by $\Lambda$, regardless of the particular semigroup $S$.

Definition 3.3. Let $\Gamma$ be the structure semigroup of $(M(S)$, *). For each $\mu \in M(\Gamma)$, define $m_{\mu}: \Lambda \rightarrow \Lambda$ by defining $m_{\mu}$ on the linear span of $\Delta(M(S))$ as

$$
\begin{aligned}
m_{\mu}\left(\sum_{i=1}^{n} c_{i} \chi_{i}\right) & =\sum_{i=1}^{n} c_{i}\left(\chi_{i}, \mu\right) x_{i}, \\
\chi_{i} & \in \Delta(M(S)), c_{i} \in C \text { for } i=1,2, \ldots, n .
\end{aligned}
$$

That is, $m_{\mu}(f)$ is the Arens product of $\mu \in M(\Gamma)=\Lambda^{*}$ and $f \in \Lambda$, and $\left\|m_{\mu}\right\| \leq\|\mu\|$.

We use the standard notation that if $T: B \rightarrow B$ is a function defined on a linear vector space $B$, then the adjoint $T^{*}: B^{*} \rightarrow B^{*}$ is defined by $T^{*}(f)=f \circ T$.

Lemma 3.4. Let $\mu, \nu \in M(\Gamma)$. Then $\left(m_{\mu}(f), \nu\right)=(f, \mu * \nu)$ for all $f \in \Lambda$. Hence, $m_{\mu}^{*}(\nu)=\mu * \nu$ for all $\nu \in M(\Gamma)$. 
Proof. For each $\chi \in \Delta(M(S))$,

$$
\left(\chi, m_{\mu}^{*}(v)\right)=\left(m_{\mu}(\chi), \nu\right)=((\chi, \mu) \chi, \nu)=(\chi, \mu)(\chi, \nu)=(\chi, \mu * v) .
$$

Hence, $\left(f, m_{\mu}^{*}(\nu)\right)=(f, \mu * \nu)$ for all $f \in \Lambda$ since the linear span of $\Delta(M(S))$ is dense in $\Lambda$. Thus, $m_{\mu}^{*}(\nu)=\mu * \nu$ for all $\nu \in M(\Gamma)$. This completes the proof.

If $M(S)$ has a weak bounded approximate identity and $T \in \mathcal{M}(M(S))$, the following calculations show that $\left.T^{*}\right|_{\Lambda}=m_{\mu_{T}}$ : if $\chi \in \Delta(M(S))$,

$$
\begin{aligned}
\left(T^{*}(\chi), \alpha\right) & =(\chi, T(\alpha))=T(\alpha)^{\wedge}(\chi) \\
& =\left(\mu_{T} * \alpha\right)^{\wedge}(\chi)=\hat{\mu}_{T}(\chi) \hat{\alpha}(\chi) \\
& =\left(\hat{\mu}_{T}(\chi) \chi, \alpha\right)=\left(m_{\mu_{T}}(\chi), \alpha\right) \quad \text { for all } \alpha \in M(S)
\end{aligned}
$$

thus, $T^{*}(\chi)=m_{\mu_{\tau}}(\chi)$ for all $\chi \in \Delta(M(S))$. Also, $T^{*}$ (and hence $m_{\mu_{\tau}}$ ) is a continuous function on $\Lambda$ in the $w(\Lambda, M(S))$ topology [19, Lemma 5.10]. We wish to investigate the converse question: that is, if $\mu \in M(\Gamma)$ is such that $m_{\mu}$ is continuous on $\Lambda$ in the $w(\Lambda, M(S))$ topology, does $\mu$ determine a multiplier of $M(S)$ ? Indeed, we show that if $\mu \in M(\Gamma)=\Lambda^{*}$ is in addition continuous on $\Lambda$ in the $w(\Lambda, M(S))$ topology, $\mu$ determines a multiplier of $M(S)$. In what immediately follows, we remove the restriction that $M(S)$ has a weak bounded approximate identity and assume only that $M(S)$ is semisimple. First, we proceed to establish some preliminary lemmas.

Recall that if $B$ is a linear vector space, $E$ is a total subspace of $B^{*}$ if $g(x)=0$ for all $g \in E$ implies that $x=0, x \in B$.

Lemma 3.5. If $E$ is a weak-* dense subspace of $B^{*}$, then $E$ is a total subspace of $B^{*}$.

Proof. Suppose $x \in B$ and $(x, g)=0$ for all $g \in E$. If $h \in B^{*}$, there exists a net $\left\{h_{\rho}\right\} \subset E$ such that $h_{\rho} \rightarrow h$ weak-*, that is $\left(y, h_{\rho}\right) \rightarrow(y, h)$ for all $y \in B$. Thus, $0=\left(x, h_{\rho}\right) \rightarrow(x, h)$ implies that $(x, h)=0$ for all $h \in B^{*}$. Therefore, $\boldsymbol{x} \equiv \mathbf{0}$. This completes the proof.

Lemma 3.5 thus implies that $p(M(S))$ is a total subspace of $\Lambda^{*}$.

Lemma 3.6. If $\mu \in M(\Gamma)$ is $w(\Lambda, M(S))$ continuous on $\Lambda$, then $m_{\mu}$ is also $w(\Lambda, M(S))$ continuous on $\Lambda$.

Proof. Suppose $f \in \Lambda,\left\{f_{\rho}\right\} \subset \Lambda$ and $f_{\rho} \rightarrow f$ in the $w(\Lambda, M(S))$ topology. If $\nu \in M(S)$ is fixed, for all $\beta \in M(S)$ we have

$$
\left(m_{\nu}\left(f_{\rho}\right), \beta\right)=\left(f_{\rho}, \nu * \beta\right) \rightarrow(f, \nu * \beta)=\left(m_{\nu}(f), \beta\right),
$$

and hence $m_{\nu}\left(f_{\rho}\right) \rightarrow m_{\nu}(f)$ in the $w(\Lambda, M(S))$ topology for each $\nu \in M(S)$. The $w(\Lambda, M(S))$ continuity of $\mu$ on $\Lambda$ implies that $\left(m_{\mu}\left(f_{\rho}\right), \nu\right)=\left(m_{\nu}\left(f_{\rho}\right), \mu\right) \rightarrow\left(m_{\nu}(f)\right.$, $\mu)=\left(m_{\mu}(f), \nu\right)$ for all $\nu \in M(S)$. Thus, $m_{\mu}\left(f_{\rho}\right) \rightarrow m_{\mu}(f)$ in the $w(\Lambda, M(S))$ topology on $\Lambda$. 
Theorem 3.7. If $\mu \in M(\Gamma)$ is such that $m_{\mu}$ is $w(\Lambda, M(S))$ continuous on $\Lambda$, then $\mu$ determines a multiplier of $M(S)$.

Proof. For each $\beta \in M(S)$, the function $f \mapsto\left(m_{\mu}(f), \beta\right), f \in \Lambda$, is continuous in the $w(\Lambda, M(S))$ topology on $\Lambda: f_{\rho} \rightarrow f$ implies $m_{\mu}\left(f_{\rho}\right) \rightarrow m_{\mu}(f)$ implies $\left(m_{\mu}\left(f_{\rho}\right), \beta\right) \rightarrow\left(m_{\mu}(f), \beta\right)$ for all $\beta \in M(S),\left\{f_{\rho}\right\} \subset \Lambda, f \in \Lambda$. By Lemma 3.5, $p(M(S))$ is a total subspace of $\Lambda^{*}$, so there exists an element $K \beta \in M(S)$ such that $\left(m_{\mu}(f), \beta\right)=(f, p(K \beta))=f(K \beta)$ for all $f \in \Lambda[7, \mathrm{~V}, 3.9] . p(K \beta)$ is linear on $\Lambda$ and

$$
\begin{aligned}
\|K \beta\| & =\|p(K \beta)\|=\sup _{f \in \Lambda ;\|f\|_{\infty} \leq 1}|(f, p(K \beta))| \\
& =\sup _{f \in \Lambda ;\|f\|_{\infty} \leq 1}\left|\left(m_{\mu}(f), \beta\right)\right| \leq\|\mu\|\|\beta\| .
\end{aligned}
$$

Now, $K$ is a linear transformation on $M(S)$ and \|\|$K\|\leq\| \mu \|$. Thus, $K$ is a bounded linear transformation on $M(S)$ with $m_{\mu}=\left.K^{*}\right|_{\Lambda}$ since $\left(m_{\mu}(f), \beta\right)$ $=f(K \beta)$ for all $\beta \in M(S)$ and for all $f \in \Lambda$. Moreover, if $\beta \in M(S)$,

$$
\begin{aligned}
(\chi, p(K \beta)) & =(\chi, j(K \beta))=\left(\chi, K^{* *} j(\beta)\right) \\
& =\left(\chi, j(\beta) \circ K^{*}\right)=\left(m_{\mu}(\chi), j(\beta)\right) \\
& =\left(m_{\mu}(\chi), p(\beta)\right)=((\chi, \mu) \chi, p(\beta)) \\
& =(\chi, \mu)(\chi, p(\beta))=(\chi, \mu * p(\beta)) \text { for all } \chi \in \Delta(M(S))
\end{aligned}
$$

Hence, for each $\beta \in M(S), p(K \beta)$ and $\mu * p(\beta)$ are the same measure in $M(\Gamma)$, and so $\mu * p(\beta) \in p(M(S))$ for all $\beta \in M(S)$. Thus, if we now identify $M(S)$ with $p(M(S)), T_{\mu}(\beta)=\mu * \beta, \beta \in M(S)$, defines a multiplier of $M(S)$. This completes the proof.

We are now in a position to characterize those measures in $M(\Gamma)$ that give rise to multipliers of $M(S)$ when $M(S)$ has a weak bounded approximate identity.

Corollary 3.8. (a) If $\mu \in M(\Gamma)$ is such that $\mu$ is $w(\Lambda, M(S))$ continuous on $\Lambda$, then $\mu$ determines a multiplier of $M(S)$.

(b) Suppose $M(S)$ has a weak bounded approximate identity. Then $T$ is a multiplier of $M(S)$ if and only if there exists a unique measure $\mu_{T} \in M(\Gamma)$ such that $m_{\mu_{T}}$ is $w(\Lambda, M(S))$ continuous on $\Lambda$. Moreover, $T$ and $\mu_{T}$ satisfy $T(\beta)=\mu_{T} * \beta$ for all $\beta \in M(S)$.

Proof. (a) Lemma 3.6 and Theorem 3.7.

(b) The remarks prior to Lemma 3.5 show that if $T \in \mathcal{M}(M(S))$, then $m_{\mu_{T}}$ is $w(\Lambda, M(S))$ continuous on $\Lambda$, where $\mu_{T}$ is the unique measure in $M(\Gamma)$ that corresponds to $T$ [Theorem 3.1]. On the other hand, if $\mu \in M(\Gamma)$ is such that $m_{\mu}$ is $w(\Lambda, M(S))$ continuous on $\Lambda$, Theorem 3.7 establishes that $\mu$ determines a multiplier of $M(S)$. 
Corollary 3.9. Assume that $M(S)$ has an identity. Suppose $g$ is a complex valued $w(\Delta(M(S)), M(S))$ continuous function on $\Delta(M(S))$ that has a linear $w(\Lambda, M(S))$ continuous extension to $\Lambda$. Then $g=\hat{\mu}$ for some $\mu \in M(S)$ if and only if there exists a constant $R$ such that $\left|\sum_{i=1}^{n} c_{i} g\left(\chi_{i}\right)\right| \leq R\|f\|_{\infty}$ for all $f=\sum_{i=1}^{n} c_{i} \chi_{i}$, $\chi_{i} \in \Delta(M(S)), c_{i} \in C, i=1,2, \ldots, n$.

Proof. If $\hat{\mu} \in M(S)^{\wedge}$, the inequality holds, where $R=\|\mu\|$; clearly, $\hat{\mu}$ has a linear $w(\Lambda, M(S))$ continuous extension to $\Lambda$. On the other hand, if $g$ has a linear extension $\bar{g}$ to $\Lambda$ and satisfies the stated inequality, $\bar{g} \in \Lambda^{*}$. The fact that $\bar{g}$ is $w(\Lambda, M(S))$ continuous on $\Lambda$ implies that $\bar{g} \in \mathcal{M}(M(S))$ by Corollary 3.8(a), and since $M(S)$ has an identity, $\bar{g}=\hat{\mu}$ for some $\mu \in M(S)$. This completes the proof.

If $T \in \mathcal{O}(M(S))$, then $\hat{T}$ (and hence $\mu_{T}$, if $M(S)$ has a weak bounded approximate identity) is $w(\Delta(M(S)), M(S))$ continuous on $\Delta(M(S))$ since $\Delta(M(S))$ is homeomorphic to an open subset of $\Delta(M(M(S)))$ [2] or [21]. Corollary 3.8(a) gives a partial converse to this result; however, we require that $\mu \in M(\Gamma)$ be $w(\Lambda, M(S))$ continuous on $\Lambda$ and not simply on $\Delta(M(S)) \subset \Lambda$ in order that $\mu$ determine a multiplier of $M(S)$. From this viewpoint there is some similarity to Eberlein's characterization of bounded continuous functions on the dual of a locally compact abelian group $G$ that are transforms of measures in $M(G)[8]$. If $G$ is a locally compact abelian topological group, the Bohr compactification $\bar{G}$ of $G$ is the structure semigroup for $L_{1}(G)$, and $M\left(L_{1}(G)\right)$ is isometrically isomorphic to $M(G)$ by Theorem 3.2. Now if $\mu \in M(G), \mu$ $\in M(\bar{G})$ and $\mu($ or $\hat{\mu})$ is $w\left(\hat{G}, L_{1}(G)\right)$ continuous on $\hat{G}$; likewise, any measure $\bar{\mu} \in M(\bar{G})$ that is $w\left(\hat{G}, L_{1}(G)\right)$ continuous on $\hat{G}$ is actually concentrated on $G$ and hence belongs to $M(G)$ [18]. In keeping with this train of thought, Corollary 3.9 characterizes those $w(\Delta(M(S)), M(S))$ continuous functions on the maximal ideal space of $M(S)$ that have $w(\Lambda, M(S))$ continuous linear extensions to $\Lambda$, in the event that $M(S)$ has an identity.

It should be pointed out that Rennison's approach [16] in arriving at the structure semigroup $\Gamma$ of $M(S)$ makes it clear that $M(\Gamma)$ is the Birtel extension [3] of $M(S)$. Birtel discusses in [3] the multiplier algebra of a Banach algebra $B$ and states several results in which he assumes the topological embedding of either $B$ or $M(B)$ into what we are now referring to as the Birtel extension of $B$. Relating the work of Taylor [20] to that of Rennison [16], it turns out that the embedding of $M(S)$ into its Birtel extension $M(\Gamma)$ is an isometry if and only if $M(S)$ is semisimple [20]. Our main contribution with regard to Birtel's work is that Theorem 3.1 with $A=M(S)$ establishes the topological embedding $T \mapsto \mu_{T}$ of $\mathcal{C}(M(S))$ into $M(\Gamma)$ with $\|T\|\left|\leq\left\|\mu_{T}\right\| \leq R\|T\|\right|$; moreover, $T \mapsto \mu_{T}$ is an isometry if and only if the weak bounded approximate identity is of norm one.

For sake of completeness we note that Corollary $3.8(\mathrm{~b})$ is analogous to Theorem 2 of [14], but the conditions are different. 
4. Multipliers of $l_{1}(S)$. Assume that $S$ is a commutative discrete semigroup. The semisimplicity of $l_{1}(S)$ is equivalent to the algebraic condition on $S$ that $x^{2}=y^{2}=x y$ implies $x=y, x, y \in S$. If $S$ satisfies this condition, we follow Petrich [15] in saying that $S$ is separative. A function $\sigma: S \rightarrow S$ having the property that $\sigma(x y)=x \sigma(y)$ for all $x, y \in S$ is called a multiplier of $S$. The set of all multipliers of $S$ is denoted $\Omega(S)$; under the operation $\circ$ of composition of functions, $\Omega(S)$ is a semigroup with identity $\bar{e}$ (though not necessarily commutative). Further, define $S$ to be reductive if $y x=z x$ for all $x \in S$ implies $y=z$. There is a natural mapping $x \mapsto \sigma_{x}$ of $S$ into $\Omega(S)$, where $\sigma_{x}(y)=x y$ for all $y \in S$. The natural mapping is one-to-one if and only if $S$ is reductive and onto if and only if $S$ has an identity. The set $\left\{\sigma_{x}: x \in S\right\} \subseteq \Omega(S)$ forms an ideal in $\Omega(S)$ and $\sigma_{x} \circ \sigma=\sigma \circ \sigma_{x}=\sigma_{\sigma(x)}$ for all $\sigma \in \Omega(S), x \in S$. For more details on these and other results on semigroups, consult the survey article [15].

The next proposition summarizes some of the relationships between $S$ and $\Omega(S)$ when given various conditions on $S$. Some of the statements in the proposition can be found in [15]; in any case, in the presence of commutativity, all are readily proved.

Proposition 4.1. (a) If $S$ has a set of relative units, then $S$ is reductive.

(b) If $S$ is reductive, then $\Omega(S)$ is commutative.

(c) If $S$ is separative, then $S$ is reductive and $\Omega(S)$ is separative.

(d) If $S$ is cancellative, then $\Omega(S)$ is cancellative, and in addition, $S$ and $\Omega(S)$ have isomorphic quotient groups.

(e) If $S$ is idempotent, then $\Omega(S)$ is idempotent.

(f) If $S$ is a union of groups, then $\Omega(S)$ is a union of groups.

Thus, we have conditions on $S$ that imply the commutativity of $\Omega(S)$, and we are able to relate the semisimplicity of $l_{1}(S)$ to that of $l_{1}(\Omega(S))$. Throughout the remainder of this section, let us assume that $\Omega(S)$ is a commutative semigroup. If we are interested in describing $\mathcal{C}\left(l_{1}(S)\right)$, then elements of $l_{1}(\Omega(S))$ determine a class of multipliers of $l_{1}(S)$.

Proposition 4.2. There is a norm-decreasing homomorphism $\tau \mapsto T_{\tau}$ of $l_{1}(\Omega(S))$ into $M\left(l_{1}(S)\right)$.

Proof. Let $\tau=\Sigma_{\sigma \in \Omega(S)} \tau(\sigma) \delta_{\sigma} \in l_{1}(\Omega(S))$ and let $P$ be the linear subspace of $l_{1}(S)$ spanned by the point masses $\left\{\delta_{2}\right\}_{z \in S}$. Define $T_{\tau}: P \rightarrow l_{1}(S)$ by $T_{\tau}\left(\delta_{2}\right)$ $=\sum_{\sigma \in \Omega(S)} \tau(\sigma) \delta_{\sigma(z)}, z \in S$, and extend linearly. $\left\|T_{\tau}\left(\delta_{z}\right)\right\| \leq \sum_{o \in \Omega(S)}|\tau(\sigma)|=\|\tau\|$ for all $z \in S$; if $\alpha=\sum_{1 \leq i \leq n} \alpha\left(z_{i}\right) \delta_{z_{i}} \in P$, then

$$
\left\|T_{\tau}(\alpha)\right\| \leq \sum_{1 \leq i \leq n}\left|\alpha\left(z_{i}\right)\right|\left\|T_{\tau}\left(\delta_{z_{1}}\right)\right\| \leq\|\tau\|\|\alpha\| .
$$

Thus, for each $\tau \in l_{1}(\Omega(S)), T_{\tau}$ is a bounded operator from $P$ into $l_{1}(S)$. The fact that $P$ is dense in $l_{1}(S)$ allows us to extend $T_{r}$ to be a bounded linear operator on $l_{1}(S)$, and indeed $\left\|T_{\tau}\right\| \leq\|\tau\|$ (no confusion arises if we not rename $T_{\tau}$ ). It is a 
routine verification to show that $T_{\tau} \in \mathcal{M}\left(l_{1}(S)\right)$ for each $\tau \in l_{1}(\Omega(S))$. Now if $S$ is reductive, $S$ is identified with a subsemigroup of $\Omega(S)$, and $T_{\tau}$ is simply convolution multiplication by $\tau \in l_{1}(\Omega(S))$.

Proposition 4.3. The natural homomorphism $g \mapsto \sigma_{g}$ of $S$ into $\Omega(S)$ induces a homomorphism of $l_{1}(S)$ into $l_{1}(\Omega(S))$ which is (a) one-to-one if and only if $x \mapsto \sigma_{x}$ is one-to-one and (b) onto if and only if $x \mapsto \sigma_{x}$ is onto.

Proof. (a) If $\alpha=\sum_{x \in S} \alpha(x) \delta_{x} \in l_{1}(S)$, the induced homomorphism of $l_{1}(S)$ into $l_{1}(\Omega(S))$ maps $\sum_{x \in S} \alpha(x) \delta_{x} \rightarrow \sum_{x \in S} \alpha(x) \delta_{\sigma_{x}}$. This map is one-to-one if and only if the set $\left\{\delta_{\sigma_{x}}\right\}$ is linearly independent, which is true if and only if $x \mapsto \sigma_{x}$ is one-to-one.

(b) If $x \mapsto \sigma_{x}$ is onto and $\sigma \in \Omega(S)$, then there exists $x \in S$ such that $\sigma=\sigma_{x}$, or in other words $\delta_{\sigma}=\delta_{\sigma_{x}}$. Conversely, if the homomorphism of $l_{1}(S)$ into $l_{1}(\Omega(S))$ is onto, then given $\sigma \in \Omega(S)$, there exists $\alpha \in l_{1}(S)$ such that $\delta_{\sigma}$ $=\sum_{x \in S} \alpha(x) \delta_{\sigma_{x}}$. If $\sigma \neq \sigma_{x}$ for all $x \in S$, then $0=\delta_{\sigma}\left(\sigma_{x}\right)=\alpha(x)$ for all $x \in S$ implies that $\delta_{\sigma} \equiv 0$. Thus, there exists $x \in S$ such that $\sigma=\sigma_{x}$ and $\sigma \mapsto \sigma_{x}$ is onto. This completes the proof.

Since the homomorphic image $\Omega$ of $l_{1}(S)$ in $l_{1}(\Omega(S))$ is an ideal in $l_{1}(\Omega(S))$, it is true that $\Delta\left(l_{1}(\Omega(S))\right) \backslash h(\Omega)$ is homeomorphic to $\Delta(\mathscr{\Omega})$ by Theorem 3.1.18 of [17], where $h(\Omega)$ is the hull of $\Omega$. For instance, if $S$ is separative, then we can consider $S$ to be a subset of $\Omega(S) ; \Omega(S)$ is commutative and separative. Moreover, $\Omega(S)^{\text {a }}$ is homeomorphic to $\hat{S}$ in union with the hull of $l_{1}(S)$.

Proposition 4.4. Suppose $S$ is a semigroup having the following property: Given $\sigma \in \Omega(S)$ there exists $x_{\sigma} \in S$ such that $\sigma\left(x_{\sigma}\right)=\sigma^{\prime}\left(x_{\sigma}\right)$ implies $\sigma \equiv \sigma^{\prime}$, where $\sigma^{\prime} \in \Omega(S)$. Then $\tau \mapsto T_{\tau}$ mapping $l_{1}(\Omega(S))$ into $\mathcal{M}\left(l_{1}(S)\right)$ is one-to-one.

Proof. Suppose $\tau=\Sigma_{\sigma \in \Omega(S)} \tau(\sigma) \delta_{\sigma}$ and $T_{\tau}\left(\delta_{x}\right)=0$ for all $x \in S, \tau \neq 0$. Choose $\sigma \in \Omega(S)$ such that $\tau(\sigma) \neq 0$. Then there exists $x_{\sigma} \in S$ such that $\sigma\left(x_{\sigma}\right)=\sigma^{\prime}\left(x_{\sigma}\right)$ implies $\sigma \equiv \sigma^{\prime}$. Therefore,

$$
0=T_{\tau}\left(\delta_{x_{0}}\right)=\tau(\sigma) \delta_{\sigma\left(x_{0}\right)}+\sum_{\sigma^{\prime} \in \Omega(S) ; \sigma^{\prime} \neq 0} \tau\left(\sigma^{\prime}\right) \delta_{\sigma^{\prime}\left(x_{0}\right)}
$$

implies that $\tau(\sigma)=0$. Thus, $\tau \mapsto T_{\tau}$ is a one-to-one map.

Corollary 4.5. If $S$ is a cancellative semigroup, then $\tau \mapsto T_{\tau}$ is one-to-one.

Proof. We verify the conditions of Proposition 4.4. For a given $\sigma \in \Omega(S)$ take $x_{\sigma}$ to be any element in $S$. Since $\Omega(S)$ is cancellative, if $\sigma \circ \sigma_{x}=\sigma^{\prime} \circ \sigma_{x}$, then it follows that $\sigma \equiv \sigma^{\prime}$. This completes the proof.

If $S$ is a semigroup with cancellation, then Proposition 4.1(d) establishes the existence of an isomorphic embedding of $S$ and $\Omega(S)$ in a group $G$ which has the property that $\Omega(S)=\{\sigma \in G: \sigma S \subset S\}$. We use this embedding and modify the arguments of Davis [6] in proving the next result. 
Theorem 4.6. If $S$ is a semigroup with cancellation, then $M\left(l_{1}(S)\right)$ is isometrically isomorphic to $l_{1}(\Omega(S))$.

Proof. $l_{1}(S)$ and $l_{1}(\Omega(S))$ are the subalgebras of $l_{1}(G)$ whose elements are supported on $S$ and $\Omega(S)$ respectively. Since $S$ is cancellative, the homomorphism $\tau \mapsto T_{\tau}$ of $l_{1}(\Omega(S))$ into $\mathcal{C M}\left(l_{1}(S)\right)$ is one-to-one by Corollary 4.5. We propose to show that it is onto. Let $T \in \mathcal{M}\left(l_{1}(S)\right)$ and let $\alpha \in l_{1}(S)$. If $x \in S$, then $T(\alpha) * \delta_{x}=\alpha * T\left(\delta_{x}\right)$; thus, $T(\alpha)=\alpha * T\left(\delta_{x}\right) * \delta_{x-1}$ for all $\alpha \in l_{1}(S)$. The interesting fact is that $T\left(\delta_{x}\right) * \delta_{x^{-1}} \in l_{1}(\Omega(S))$. For suppose there exists $y \in G, z \in S$ such that $y z \notin S$ and $\left[T\left(\delta_{x}\right) * \delta_{x^{-1}}\right](y) \neq 0$. Then $\left[\delta_{z} * T\left(\delta_{x}\right) * \delta_{x^{-1}}\right](y z)=$ $\left[T\left(\delta_{x}\right) * \delta_{x^{-1}}\right](y) \neq 0$ and hence $\delta_{z} * T\left(\delta_{x}\right) * \delta_{x^{-1}} \notin l_{1}(S)$. This contradicts the fact that $\alpha * T\left(\delta_{x}\right) * \delta_{x^{-1}} \in l_{1}(S)$ for all $\alpha \in l_{1}(S)$. Thus, given $T \in \mathcal{M}\left(l_{1}(S)\right)$ there exists $\tau \in l_{1}(\Omega(S))$ such that $T(\alpha)=\alpha * \tau=T_{\tau}(\alpha)$ for all $\alpha \in l_{1}(S)$ and hence $\tau \mapsto T_{\tau}$ is an onto map. From Proposition 4.2 we know that $\left\|T_{\tau}\right\| \leq\|\tau\|$ for all $\tau \in l_{1}(\Omega(S))$. But

$$
\left\|T_{\tau}\right\| \mid=\sup _{\alpha \in l_{1}(S) ;\|\alpha\|=1}\|\tau * \alpha\|>\left\|\tau * \delta_{x}\right\|=\|\tau\|,
$$

and hence $\left|\left\|T_{\tau}\right\|\right|=\|\tau\|$. Therefore, we have proved that $c M\left(l_{1}(S)\right)$ is isometrically isomorphic to $l_{1}(\Omega(S))$. This completes the proof.

For the remainder of this section we assume that $l_{1}(S)$ is semisimple, and that $l_{1}(S)$ has a weak bounded approximate identity of norm one. This is equivalent to the existence of an identity in the structure semigroup $\Gamma$ of $\left(l_{1}(S), *\right)$ by Corollary 3.2 of [11]. In Proposition 4.1 of [11] $S$ is realized as a dense subsemigroup of $\Gamma$ by the isomorphism $i_{s}: S \rightarrow \Gamma$. Let us consider the question of embedding $\Omega(S)$ in $\Gamma$. If $\Gamma$ does not have an identity, then clearly there can be no isomorphic embedding of $\Omega(S)$ in $\Gamma$ which extends the natural map $i_{s}$ of $S$ into $\Gamma$, since an identity for $i_{s}(S)$ is an identity for $\Gamma$. As the next theorem shows, the presence of an identity in $\Gamma$ is sufficient to guarantee the embedding of $\Omega(S)$ in $\Gamma$.

Theorem 4.7. If $S$ is separative, the natural isomorphism $i_{s}$ of $S$ into $\Gamma$ has an extension to an isomorphism $i_{\Omega(S)}$ of $\Omega(S)$ into $\Gamma$ if and only if $\Gamma$ has an identity.

Proof. If $i_{\Omega(S)}$ is an isomorphism of $\Omega(S)$ into $\Gamma$ and extends the natural isomorphism $i_{s}, \Gamma$ has an identity since $i_{s}(S)$ is $w(\Gamma, C(\Gamma))$-dense in $\Gamma[11$, Proposition 4.1] and $\Omega(S)$ has identity $\bar{e}$.

On the other hand, assume $\Gamma$ has an identity $e$, and let $i_{s}(x)=\tilde{x}$ for each $x \in S$. By Proposition 4.1 of [11], there is a net $\left\{\tilde{u}_{d}\right\} \subset i_{s}(S)$ such that $\tilde{u}_{d} \rightarrow e$. Fix $\sigma \in \Omega(S)$ and consider $\left\{\sigma\left(u_{d}\right)^{\sim}\right\} \subset \Gamma$. Since $\Gamma$ is compact, there exists a subnet $\left\{\sigma\left(u_{d^{\prime}}\right)^{\sim}\right\}$ and $\tilde{\sigma} \in \Gamma$ such that $\sigma\left(u_{d^{\prime}}\right)^{\sim} \rightarrow \tilde{\sigma} ;$ thus, $\tilde{\sigma} \cdot \tilde{x}=\lim \tilde{x} \sigma\left(u_{d^{\prime}}\right)^{\sim}$ $=\lim \sigma(x)^{\sim} \tilde{u}_{d^{\prime}}=\sigma(x)^{\sim}$ for all $x \in S$. Now, let $\left\{\tilde{x}_{\rho}\right\} \subset i_{s}(S)$ be any net such that $\tilde{x}_{\rho} \rightarrow e$ and suppose that in accordance with the compactness of $\Gamma$ there is a subnet $\sigma\left(x_{\rho^{\prime}}\right)^{\sim} \rightarrow \tilde{\tau} \in \Gamma$; then $\tilde{\sigma} \cdot \tilde{x}=\sigma(x)^{\sim}=\tilde{\tau} \cdot \tilde{x}$ for all $x \in S$ implies that for each $\chi \in \hat{\Gamma}, \chi(\tilde{\sigma})=\chi(\tilde{\tau})$. Indeed, given $\chi \in \hat{\Gamma}$, choose $z \in S$ such that $\chi(\tilde{z}) \neq 0$, in which case we have that 


$$
\chi(\tilde{\sigma}) \chi(\tilde{z})=\chi(\tilde{\sigma} \cdot \tilde{z})=\chi(\tilde{\tau} \cdot \tilde{z})=\chi(\tilde{\tau}) \chi(\tilde{z}) ;
$$

since $\hat{\Gamma}$ separates points of $\Gamma[20]$, it must be that $\tilde{\sigma}=\tilde{\tau}$. Thus, multiplication by $\tilde{\sigma} \in \Gamma$ determines an element of $\Omega(S)$. We may now define $i_{\Omega(S)}: \Omega(S) \rightarrow \Gamma$ by $i_{\Omega(S)}(\sigma)=\tilde{\sigma}$ for each $\sigma \in \Omega(S)$. $i_{\Omega(s)}$ is one-to-one because $i_{Q(S)}(\sigma)=\tilde{\sigma}$ $=i_{\Omega(S)}(\tau), \sigma, \tau \in \Omega(S)$ implies that $\sigma(x)^{\mathcal{L}}=\tilde{\sigma} \cdot \tilde{x}=\tau(x)^{\sim}$ for all $x \in S$ and so $\sigma \equiv \tau$. This completes the proof.

Now that we have an embedding of $\Omega(S)$ in $\Gamma, l_{1}(\Omega(S))$ can be identified with a subalgebra of $l_{1}(\Gamma) \subseteq M(\Gamma)$. Note that this means that $\hat{\tau}(\chi)=0$ for all $\chi \in \hat{S}$ implies $\tau \equiv 0, \tau \in l_{1}(\Omega(S))$. Also, Theorem 3.1 implies that the embedding $T \mapsto \mu_{T}$ of $\mathcal{M}\left(l_{1}(S)\right)$ in $M(\Gamma)$ is an isometry, in which case we have $\left\|T_{\tau}\right\| \mid=\|\tau\|$ for all $\tau \in l_{1}(\Omega(S))$. Thus, $\left\{T_{\tau}: \tau \in l_{1}(\Omega(S))\right\}$ is an operator-norm closed subalgebra of $\mathcal{M}\left(l_{1}(S)\right)$. This leads to the conjecture that $\mathcal{M}\left(l_{1}(S)\right)$ is isometrically isomorphic to $l_{1}(\Omega(S))$. We propose to use the representation theory for multipliers of semisimple convolution measure algebras with weak bounded approximate identities developed in $\$ 3$ in order to show that this is true. The measure $\mu \in M(\Gamma)$ is a multiplier of $l_{1}(S)$ if and only if $m_{\mu}$ is $w\left(\Lambda, l_{1}(S)\right)$ continuous on $\Lambda$ by Corollary 3.8(b). Our aim is to show that if $\mu \in M(\Gamma)$ is such that $m_{\mu}$ is $w\left(\Lambda, l_{1}(S)\right)$ continuous on $\Lambda$, then indeed $\mu \in l_{1}(\Omega(S))$.

The fact that $\Omega(S)$ can be considered a subset of $\Gamma$ affords us a new way of relating the semicharacters of $S$ to their extensions in $\Omega(S)^{\star}$. Each $\chi \in \hat{S}$ has a unique extension $\tilde{\chi} \in \Omega(S)^{\wedge}$. Now $\Lambda$ is isometrically isomorphic to $C(\Gamma)$ with the mapping denoted $g \mapsto g^{\prime}$; therefore, it must be that for each $\chi \in \hat{S},\left.\chi^{\prime}\right|_{\Omega(S)}=\tilde{\chi}$. Also, there is a one-to-one correspondence between the linear span of $S$ in $l_{1}(S)^{*}=l_{\infty}(S)$ and the linear span $L$ of $\left\{\tilde{\chi} \in \Omega(S)^{*}: x \in S\right\} \subset l_{1}(\Omega(S))^{*}$ $=l_{\infty}(\Omega(S))$ by mapping

$$
\left.\sum_{i=1}^{n} c_{i} x_{i} \mapsto \sum_{i=1}^{n} c_{i} x_{i}^{\prime}\right|_{(x)}
$$

The next proposition relates $\Lambda$ to the closure $\bar{L}$ of $L$ in $l_{\infty}(\Omega(S))$.

Proposition 4.8. There is a one-to-one correspondence between $\Lambda$ and the uniform closure of $L$ in $l_{1}(\Omega(S))^{*}$.

Proof. We have the following situation: $S \subset \Omega(S) \subset \Gamma$. Now if a sequence $\left\{\tilde{f}_{n}\right\} \subset L$ converges to $\tilde{f} \in \bar{L}$ uniformly on $\Omega(S)$, then certainly the sequence $\left\{\left.\tilde{f}_{n}\right|_{s}\right\}$ converges to $\left.\tilde{f}\right|_{s} \in \Lambda$ uniformly on $S$. The interesting point to recognize is that since uniform convergence on $S$ and on $\Gamma$ are equivalent, then uniform convergence of a sequence $\left\{f_{n}\right\}$ of elements derived from the linear span of $S$ implies uniform convergence in $l_{\infty}(\Omega(S))$ of the sequence whose elements are extensions of $f_{n}$ to $\tilde{f}_{n} \in L$ for all $n$.

Theorem 4.9. Suppose $l_{1}(S)$ has a weak bounded approximate identity of norm one. If $\mu \in M(\Gamma)=\Lambda^{*}$ is such that $m_{\mu}$ is $w\left(\Lambda, l_{1}(S)\right)$ continuous on $\Lambda$, then 
convolution multiplication by $\mu$ determines a multiplier of $l_{1}(\Omega(S))$. Hence, $M\left(l_{1}(S)\right)$ is isometrically isomorphic to $l_{1}(\Omega(S))$.

Proof. We may extend the isometry $p: l_{1}(S) \rightarrow M(\Gamma)$ in a natural way to $\bar{p}: l_{1}(\Omega(S)) \rightarrow M(\Gamma)$. The mapping $\bar{p}$ is induced by the embedding of $\Omega(S)$ in $\Gamma$, and indeed, $\bar{p}$ is an isometry. In what follows we may identify $\Lambda$ with a closed subset of $l_{1}(\Omega(S))^{*}$ by Proposition 4.8; we also recognize that $\Lambda=C(\Gamma)$. Viewing $\Lambda$ as a subset of $l_{1}(\Omega(S))^{*}$, note that $(f, \bar{p}(\tau))=f(\tau)$ for all $\tau \in l_{1}(\Omega(S))$, $f \in \Lambda$. Let $\mu \in M(\Gamma)$ be such that $m_{\mu}$ is $w\left(\Lambda, l_{1}(S)\right)$ continuous on $\Lambda$. Corollary 3.8(b) implies that $\mu$ determines a multiplier of $l_{1}(S)$. Now, $m_{\mu}$ is $w\left(\Lambda, l_{1}(\Omega(S))\right)$ continuous on $\Lambda$. Thus, for each $\tau \in l_{1}(\Omega(S))$, the function $f \mapsto\left(m_{\mu}(f), \tau\right)$, $f \in \Lambda$, is continuous on $\Lambda$ in the $w\left(\Lambda, l_{1}(\Omega(S))\right)$ topology, and is also linear. Since $p\left(l_{1}(S)\right) \subset \bar{p}\left(l_{1}(\Omega(S))\right)$, by Lemma 3.5, $\bar{p}\left(l_{1}(\Omega(S))\right)$ is a total subspace of $\Lambda^{*}$; so there exists $K \tau \in l_{1}(\Omega(S))$ such that $\left(m_{\mu}(f), \tau\right)=(f, \bar{p}(K \tau))=f(K \tau)$ for all $f \in \Lambda[7, \mathrm{~V}, 3.9]$. Now $\bar{p}(K \tau)$ is linear on $\Lambda$ and

$$
\begin{aligned}
\|K \tau\| & =\|\bar{p}(K \tau)\|=\sup _{f \in \Lambda ;\|f\| \leq 1}|(f, \bar{p}(K \tau))| \\
& =\sup _{f \in \Lambda ;\|f\| \leq 1}\left|\left(m_{\mu}(f), \tau\right)\right| \leq\|\mu\|\|\tau\| .
\end{aligned}
$$

Also, $K$ is a linear transformation on $l_{1}(\Omega(S))$ and $\|K\|\|\leq\| \mu \|$. Since $\left(m_{\mu}(f), \tau\right)$ $=f(K \tau)$ for all $\tau \in l_{1}(\Omega(S))$ and for all $f \in \Lambda, m_{\mu}=\left.K^{*}\right|_{\Lambda}$. Moreover, if $\tau \in l_{1}(\Omega(S))$,

$$
\begin{aligned}
(\chi, \bar{p}(K \tau)) & =(\chi, j(K \tau))=\left(\chi, K^{* *} j(\tau)\right) \\
& =\left(\chi, j(\tau) \circ K^{*}\right)=\left(m_{\mu}(\chi), j(\tau)\right) \\
& =\left(m_{\mu}(\chi), \bar{p}(\tau)\right)=((\chi, \mu) \chi, \bar{p}(\tau)) \\
& =(\chi, \mu)(\chi, \bar{p}(\tau))=(\chi, \mu * \bar{p}(\tau))
\end{aligned}
$$

for all $\chi \in \hat{S}$. Hence, for each $\tau \in l_{1}(\Omega(S)), \bar{p}(K \tau)$ and $\mu * \bar{p}(\tau)$ are the same measure in $M(\Gamma)$. Therefore, $T_{\mu}(\tau)=\mu * \tau$, for all $\tau \in l_{1}(\Omega(S))$, defines a multiplier of $l_{1}(\Omega(S))$, where we have identified $l_{1}(\Omega(S))$ with $\bar{p}\left(l_{1}(\Omega(S))\right)$. Because $l_{1}(\Omega(S))$ has an identity, then $\mu \in l_{1}(\Omega(S))$. Finally, we have proved that every multiplier $\mu$ of $l_{1}(S)$ is actually an element of $l_{1}(\Omega(S))$. Thus, $M\left(l_{1}(S)\right)$ is isometrically isomorphic to $l_{1}(\Omega(S))$. This completes the proof.

We conclude this paper with an example. At this point one might conjecture that every multiplier of $l_{1}(S)$ is of the form $T_{\text {r }}$ for some $\tau \in l_{1}(\Omega(S))$. However, this is not always true, as Example 4.10 shows.

Example 4.10. Let $S=\{0,1,2, \ldots, n, \ldots\}$ under the operation $n^{2}=n$ for all $n$ and $n m=0$ for $n \neq m$. It can be shown that $\Omega(S)=\{F: F$ is a subset of $S\}$ under the semigroup operation of union of sets, where each $F \subset S$ gives rise to a multiplier of $S$ by mapping 


$$
\begin{array}{ll}
n \mapsto 0, & \text { if } n \in F \text { or } n=0, \\
n \mapsto n, & \text { if } n \notin F .
\end{array}
$$

It is also a routine matter (as indicated in [10]) to verify that there is a one-toone correspondence between the filters of $\{F\}_{F \subset S}$ and the semicharacters of $\Omega(S)$, each filter being a set where a semicharacter assumes the value 1 . The semicharacters $\chi_{n}$ of $S$ correspond to all the filters composed of singletons $\{n\}, n \neq 0$ (together with the function 1). We propose to determine $\Lambda$, and exhibit the structure semigroup $\Gamma$ corresponding to $l_{1}(S)$. We also show that the operator norm of a multiplier $T$ on $l_{1}(S)$ is equivalent to the supremum norm of the corresponding continuous function $g_{T}$ on $\hat{S}$.

If we observe that $\sum_{i=0}^{n} c_{i} \chi_{i}$ corresponds to the sequence $c_{0}, c_{0}+c_{1}, c_{0}+c_{2}$, $\ldots, c_{0}+c_{n}, c_{0}, \ldots, c_{0}, \ldots$, where $c_{i} \in C, i=0,1,2, \ldots, n$, then it is fairly clear that the closed linear span of $\hat{S}$ in $l_{\infty}(S)$ is the space of all convergent sequences in $l_{\infty}(S)$.

Let $\Gamma$ denote the semigroup $S$ topologized in the following manner: $S \backslash\{0\}$ retains the discrete topology of $S$; neighborhoods of 0 consist of 0 in union with all but a finite number of points of $S$. Then $\Gamma$ is compact, and an application of the Stone-Weierstrass theorem shows that $\Lambda=C(\Gamma)$. Note that no new points have been added to $S$ in order to obtain $\Gamma$. Clearly, $\hat{S}=\hat{\Gamma}$. Finally, $\Gamma$ is Taylor's structure semigroup associated with $l_{1}(S)$ since topologically the maximal ideal space of $\Lambda=C(\Gamma)$ is $\Gamma$, and there is only one semigroup product on $\Gamma$ that makes the Gelfand transforms of elements of $\hat{S}$ into semicharacters on $\Gamma$ [16].

Let us turn now to a discussion of $M\left(l_{1}(S)\right)$. If $T \in \mathcal{M}\left(l_{1}(S)\right)$ and $j \in S$,

$$
T\left(\delta_{0}\right)=T\left(\delta_{0}\right) * \delta_{j}=\delta_{0} * T\left(\delta_{j}\right)=t_{00} \delta_{0}
$$

for some $t_{00} \in C$. Likewise, $T\left(\delta_{j}\right)=\delta_{j} * T\left(\delta_{j}\right)$ implies there exists $t_{j j} \in C$ such that

$$
T\left(\delta_{j}\right)=\left(t_{00}-t_{j j}\right) \delta_{0}+t_{j j} \delta_{j} \quad \text { for all } j \in S .
$$

Since $l_{1}(S)$ is semisimple, $T$ is a bounded linear transformation on $l_{1}(S)$. Hence, $\left\|T\left(\delta_{j}\right)\right\|=\left|t_{00}-t_{j j}\right|+\left|t_{j j}\right|$ implies that there exists a natural number $R$ such that $\left|t_{j j}\right| \leq R$ for all $j=0,1,2, \ldots$ Thus, $T \in \mathcal{M}\left(l_{1}(S)\right)$ implies that $T$ has the matrix representation

$$
T \leftrightarrow\left[\begin{array}{cccc}
t_{00} & t_{00}-t_{11} & t_{00}-t_{22} & \cdots \\
0 & t_{11} & 0 & \cdots \\
0 & 0 & t_{22} & \cdots \\
0 & 0 & 0 & \cdots \\
\cdot & \cdot & \cdot & \\
\cdot & . & . & \\
. & . & . &
\end{array}\right]=\left[t_{i j}\right]_{i, j=0}^{\infty}
$$

where $\left|t_{i j}\right| \leq R$ for all $j$. 
It is also fairly easy to see that every bounded linear transformation $T: l_{1}(S)$ $\rightarrow l_{1}(S)$ which has such a matrix representation, with $\left|t_{i i}\right| \leq R$ for all $i$ and some natural number $R$, must be a multiplier of $l_{1}(S)$. In this way a one-to-one correspondence is established between $\mathcal{S}\left(l_{1}(S)\right)$ and $l_{\infty}(S)$. We now want to show that this correspondence is also a topological one.

If $T \in \mathcal{M}\left(l_{1}(S)\right)$, then let $g_{T}$ be the continuous function on $S$ corresponding to $T$ [21]. It is proved in [21] that $\left\|g_{T}\right\|_{b_{0}} \leq\|T\|$. Let $\alpha=\sum_{i=0}^{\infty} \alpha(i) \delta_{i},\|\alpha\| \leq 1$. Then,

$$
\begin{aligned}
\|T(\alpha)\| & \leq \sum_{i=0}^{\infty}|\alpha(i)|\left\|T\left(\delta_{i}\right)\right\| \leq \sup _{i}\left\|T\left(\delta_{i}\right)\right\| \\
& =\sup _{i}\left\{\left|t_{00}-t_{i i}\right|+\left|t_{i i}\right|\right\} .
\end{aligned}
$$

Also, $g_{T}\left(\chi_{0}\right)=T\left(\delta_{0}\right)^{\wedge}\left(\chi_{0}\right)=\left(t_{00} \delta_{0}\right)^{\wedge}\left(\chi_{0}\right)=t_{00}$, while for all $j \neq 0$,

$$
\begin{aligned}
g_{T}\left(\chi_{j}\right) & =T\left(\delta_{j}\right)^{\wedge}\left(\chi_{j}\right) / \hat{\delta}_{j}\left(\chi_{j}\right)=T\left(\delta_{j}\right)^{\wedge}\left(\chi_{j}\right) \\
& =\left[\left(t_{00}-t_{i j}\right) \delta_{0}+t_{i j} \delta_{j}\right]^{\wedge}\left(\chi_{j}\right)=t_{i j}
\end{aligned}
$$

Thus, $\left|t_{00}-t_{i i}\right|+\left|t_{i i}\right| \leq\left|t_{00}\right|+2\left|t_{i i}\right| \leq 3\left\|g_{T}\right\|_{\infty}$ for all $i$. Therefore,

$$
\|T(\alpha)\| \leq \sup _{i}\left\{\left|t_{00}-t_{i i}\right|+\left|t_{i i}\right|\right\} \leq 3\left\|g_{T}\right\|_{\infty}
$$

for all $\alpha,\|\alpha\| \leq 1$. Combining inequalities we find that

$$
\left\|g_{T}\right\|_{\infty} \leq\|T\| \mid \leq 3\left\|g_{T}\right\|_{\infty} .
$$

This shows that \|\|$\cdot \| \mid$ on $\mathcal{M}\left(l_{1}(S)\right)$ is equivalent to $\|\cdot\|_{\infty}$ on $\left\{g_{T} \in C(\hat{S}): T\right.$ $\left.\in \mathcal{M}\left(l_{1}(S)\right)\right\}$. Thus, we have now proved that $\mathcal{M}\left(l_{1}(S)\right)$ is topologically isomorphic to $l_{\infty}(S)$ by noting that there is a one-to-one correspondence between $S$ and $\hat{S}$.

Our next step is to exhibit an element of $M\left(l_{1}(S)\right)$ that is not in $l_{1}(\Omega(S))$. Let $\sum_{n=1}^{\infty} a_{n}$ be a nonabsolutely convergent series of real numbers with $\left|a_{n}\right| \leq 1$ for all $n$; let $T$ be given by the matrix representation

$$
\left[\begin{array}{cccc}
\sum_{n=1}^{\infty} a_{n} & \sum_{n \neq 1} a_{n} & \sum_{n \neq 2} a_{n} & \cdots \\
0 & a_{1} & 0 & \cdots \\
0 & 0 & a_{2} & \cdots \\
\cdot & \cdot & 0 & \cdots
\end{array}\right]
$$

Then $T \in \mathcal{O}\left(l_{1}(S)\right)$. We wish to show that $g_{T} \neq \hat{\tau}$ for any $\tau \in l_{1}(\Omega(S))$. If $g_{T}$ is the transform of an element of $l_{1}(\Omega(S))$, then Corollary 3.9 implies that there exists a constant $R$ such that 


$$
\left|\sum_{i=0}^{N} c_{i} g_{T}\left(\chi_{i}\right)\right| \leq R\|f\|_{\infty} \quad \text { for all } f=\sum_{i=0}^{N} c_{i} \chi_{i}, \chi_{i} \in \hat{S}, c_{i} \in C .
$$

Let $R^{\prime}>R$; there exists a rearrangement $\sum a_{m_{n}}$ with partial sums $S_{n}^{\prime}$ such that $\lim _{n} S_{n}^{\prime}=R^{\prime}$. Hence, there exists $N$ such that $\left|S_{N}^{\prime}-R^{\prime}\right|<\left|R-R^{\prime}\right|$; set $f$ $=\sum_{n-1}^{N} \chi_{m_{n}}$, with $g_{T}\left(\chi_{m_{n}}\right)=a_{m_{n}}$. Hence, $\|f\|_{\infty}=1$ and $\left|\sum_{n=1}^{N} g_{T}\left(\chi_{m_{n}}\right)\right|$ $=\left|\sum_{n-1}^{N} a_{m_{n}}\right|>R\|f\|_{\infty}$, a contradiction. Thus, $g_{T} \notin l_{1}(\Omega(S))^{\wedge}$.

However, although $l_{1}(\Omega(S))^{\wedge} \neq \mathcal{C}\left(l_{1}(S)\right)$, it is of interest that $l_{1}(\Omega(S))^{\wedge}$ is dense in $l_{\infty}(\hat{S})$. First we recall [9] that if $B$ is a Banach algebra with identity with the property that $\operatorname{Re}(\hat{B})$ is sup norm dense in $C_{R}(\Delta(B))$, then $\hat{B}$ contains the characteristic function of every open-closed subset of $\Delta(B)$. Thus, if $S$ is a union of groups, then $l_{1}(\Omega(S))^{\wedge}$ contains the characteristic function of every openclosed subset of $\Omega(S)^{\wedge}[5]$.

Proposition 4.11. Suppose $S$ is a union of groups. If $\hat{S}$ has the discrete topology, then $l_{1}(\Omega(S))^{\wedge} I_{S}$ is uniformly dense in $l_{\infty}(\hat{S})$.

Proof. Since $\hat{S}$ is homeomorphic to a subset of $\Omega(S)^{\wedge}$ and $\hat{S}$ is discrete, the remarks in the above paragraph imply that $l_{1}(\Omega(S))^{\wedge}$ contains the characteristic function of every subset of $S$. This fact is enough to conclude that $\left.l_{1}(\Omega(S))^{\wedge}\right|_{S}$ is uniformly dense in $l_{\infty}(\hat{S})$. This completes the proof.

Now, in Example 4.10 a routine verification proves that $S$ has the discrete topology, and $\|\cdot\| \cdot \|$ on $\mathcal{C}\left(l_{1}(S)\right)$ is equivalent to $\|\cdot\|_{\infty}$. Thus, as an operator subalgebra of $M\left(l_{1}(S)\right), l_{1}(\Omega(S))$ is operator-norm dense in $M\left(l_{1}(S)\right)$.

The author wishes to thank Professot Lawrence $J$. Lardy for his guidance during the preparation of this paper. These results comprise a portion of the author's doctoral dissertation written while attending Syracuse University, Syracuse, New York.

\section{REFERENCES}

1. R. Arens, Operations induced in function classes, Monatsh. Math. 55 (1951), 1-19. MR 13, 372.

2. F. T. Birtel, Banach algebras of multipliers, Duke Math. J. 28 (1961), 203-211. MR 24 \#A2863.

3.- On a commutative extension of a Banach algebra, Proc. Amer. Math. Soc. 13 (1962), 815-822. MR 31 \#624.

4. P. Civin and B. Yood, The second conjugate space of a Banach algebra as an algebra, Pacific J. Math. 11 (1961), 847-870. MR 26 \#622.

5. W. W. Comfort, The Šlov boundary induced by a certain Banach algebra, Trans. Amer. Math. Soc. 98 (1961), 501-517. MR 22 \#11282.

6. T. A. Davis, The Wiener-Pitt phenomenon on semigroups, Proc. Cambridge Philos. Soc. 59 (1963), 11-24. MR 26 \#1701.

7. N. Dunford and J. T. Schwartz, Linear operations. I: General theory, Pure and Appl. Math., vol. 7, Interscience, New York, 1958. MR 22 \#8302.

8. W. F. Eberlein, Characterizations of Fourier-Stieltjes transforms, Duke Math. J. 22 (1955), 465-468. MR 17, 281.

9. T. W. Gamelin, Uniform algebras, Prentice-Hall, Englewood Cliffs, N.J., 1969.

10. E. Hewitt and H. S. Zuckerman, The $l_{1}$-algebra of a commutative semigroup, Trans. Amer. Math. Soc. 83 (1956), 70-97. MR 18, 465. 
11. C. D. Lahr, Approximate identities for convolution measure algebras, Pacific. J. Math. 47 (1973) (to appear).

12. L. J. Lardy, On the identity in a measure algebra, Proc. Amer. Math. Soc. 19 (1968), 807-810. MR 37 \#3366.

13. R. Larsen, The multiplier problem, Springer-Verlag, Berlin and New York, 1969.

14. L. Máté, Embedding multiplier operators of a Banach algebra $B$ into its second conjugate space $B^{* *}$, Bull. Acad. Polon. Sci. Sér. Sci. Math. Astronom. Phys. 13 (1965), 809-812. MR 33 \#587.

15. M. Petrich, The translation hull in semigroups and rings, Semigroup Forum 1 (1970), no. 4, 283-360. MR 42 \#1919.

16. J. F. Rennison, Arens products and measure algebras, J. London Math. Soc. 44 (1969), 369-377. MR 38 \#6367.

17. C. E. Rickart, General theory of Banach algebras, The Univ. Series in Higher Math., Van Nostrand, Princeton, N.J., 1960. MR 22 \#5903.

18. W. Rudin, Fourier analysis on groups, Interscience Tracts in Pure and Appl. Math., no. 12, Interscience, New York, 1962. MR 27 \#2808.

19. J. D. Stafney, Arens multiplication and convolution, Pacific J. Math. 14 (1964), 1423-1447. MR 31 \# 1321.

20. J. L. Taylor, The structure of convolution measure algebras, Trans. Amer. Math. Soc. 119 (1965), 150-166. MR 32 \#2932.

21. J. K. Wang, Multipliers of commutative Banach algebras, Pacific J. Math. 11 (1961), 1131-1149. MR 25 \# 1462.

22. J. G. Wendel, Left centralizers and isomorphisms of group algebras, Pacific J. Math. 2 (1952), 251-261. MR 14, \#246.

Bell Telephone laboratories, Holmdel, New Jersey 07733

Current address: Department of Mathematics, Savannah State College, Savannah, Georgia 31404 